\title{
Dynamic Curving Simulation of Tilting Train*
}

\author{
Jing ZENG** and Ren LUO** \\ **State Key Laboratory of Traction Power \\ Southwest Jiaotong University, Chengdu, China \\ E-mail: zeng@swjtu.cn
}

\begin{abstract}
The application of carbody tilting technology is the most efficient way to raise train speed during curve negotiations. This paper mainly deals with the dynamic performance simulation of the tilting train. Through the establishment of the nonlinear mathematical model for the titling train electromechanical coupled system, the carbody tilting control law, bogie radial steering mechanism, and titling train curving performance are investigated. The effect of time delay caused by the sensing and control system on the tilting performance of the train is analyzed, and the compensation methods for the time delay effect are studied.
\end{abstract}

Key words: Tilting Train, Curving Behavior, Control Law, Time Delay, Compensation

\section{Introduction}

Although China has conducted the speed-up activity of railways six times in recent ten years, and achieved good economic benefits, there still are many existing lines that are difficult to further raise speeds, especially the mountain lines with sharp curves. Together with the rapid development of modern control and computer technologies, the active suspension control technologies have been increasingly applied in railway vehicle systems in order to improve the dynamic performance $[1,2]$. Tilting train is one of the examples of active secondary suspensions with carbody tilting control during curve negotiation. Tilting the carbody inwards on curve can reduce the unbalanced lateral acceleration experienced by passengers, which can permit higher speeds and provide a variety of operational benefits [3-7]. Therefore, the application of carbody tilting technology is an efficient way to increase train speed and to shorten traveling time for existing railways with many sharp curves. Tilting trains have been operated successfully in many countries such as Italy, Spain, Germany, Sweden, Japan and so on. In China, the Ministry of Railways has also set up projects to develop tilting trains.

The main purpose of the tilting train is to reduce the uncompensated lateral acceleration felt by passengers through carbody tilting when the train negotiates the curved track. In order to achieve the best results in terms of running safety and passenger comfort, the dynamic performance of the tilting train must be carefully studied under the profiles of kinematics, control strategy and real behavior. In this paper, a mathematical model of tilting train is set up. The lateral acceleration of the leading bogie of the head car in the train set is used as the control signal of the tilting system. Then the curving behavior of the tilting train including the carbody tilting control strategy and time delay compensation, bogie radial steering mechanism is investigated.

\section{Tilting train model}

A tilting train with three cars is taken into account. The four swing rods mechanism is 
used for the tilting bogie which is located between the bogie frame and tilting bolster. The weight of the carbody and bolster is supported by the cross beams of the bogie frame through the swing rods. The electromechanical actuator is mounted between the bogie frame and the bolster, and the carbody is seated on the bolster through the air springs. According to the generated tilting control signal based on the measured curving information, the electromechanical actuator pushes the bolster and further drives the carbody to tilt a certain angle. The dynamic model of the vehicle rigid body system coupled with the control system for the tilting train is established, in which the bogie tilting mechanism, radial steering device, actuator, curving signal measurement and tilting control are considered.

The dynamic model of the tilting car with 34 degrees of freedom is set up. The degrees of freedom are longitudinal, lateral, vertical, roll, pitch and yaw motions of carbody and each bogie frame, longitudinal, lateral, yaw and spin motions of each wheelset. The motion of the tilting bolster is also considered, which is related to the controlled actuator displacement. Then the model of the train set with three cars is built by considering the coupler forces between cars, and the traction force and resistant force. During the modeling process, various kinds of nonlinear factors are considered, including nonlinear wheel/rail contact geometry, nonlinear wheel/rail creep forces, nonlinear coupler force and nonlinear secondary suspension forces. The schematic diagram of the tilting car is shown as Fig.1. The coupler and draft gear are modeled by Fig.2, in which $F_{c g}$ indicates the coupler force, $F_{1}, F_{2}, F_{3}$ and $F_{4}$ the draft gear forces. The hysteresis force and the effect of carbody tilting are considered for the draft gear.

The general equation of the tilting train is written as:

$$
M \ddot{x}+C \dot{x}+K x=F(x, \dot{x}, t)+G(t)
$$

where $M, C, K$ indicate the mass, damping and stiffness matrices respectively, $x$ the displacement vector, $F$ the nonlinear force vector including the nonlinear suspension forces, wheel/rail interaction forces, coupler forces, actuator forces, etc., and $G$ the track inputs.

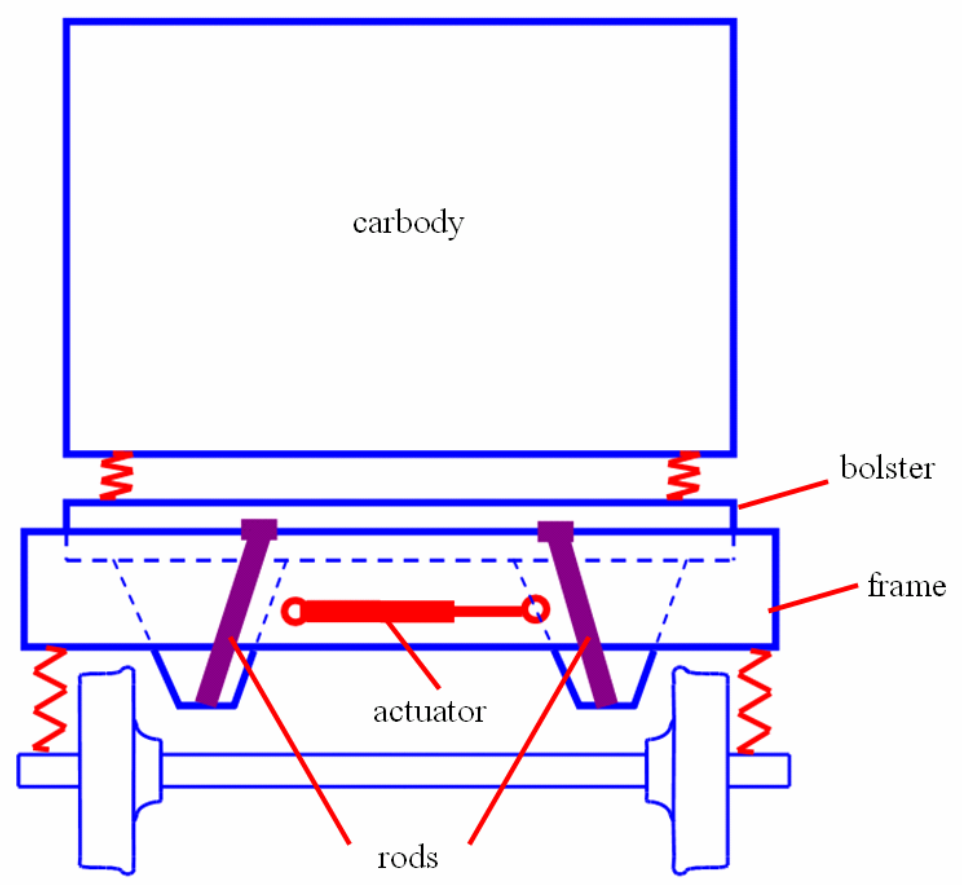

Fig.1 Schematic diagram of tilting car 


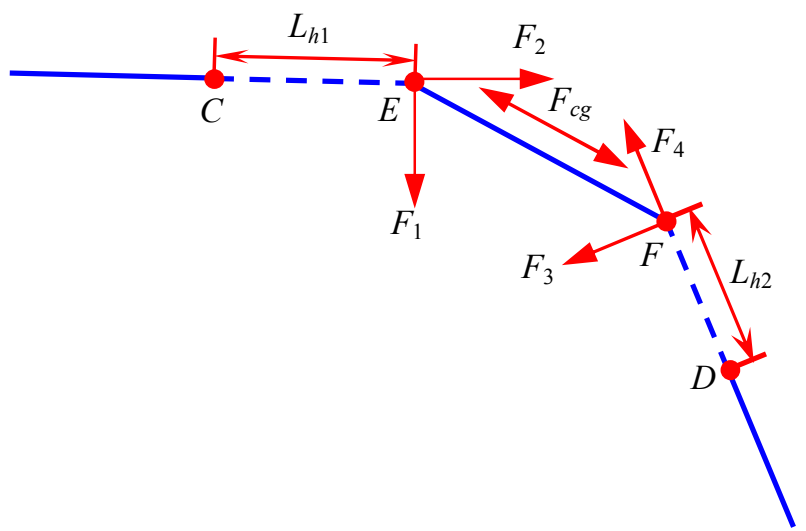

Fig.2 Forces of coupler and draft gear

In order to improve the curing behavior and reduce the wheel/rail wear for the tilting train, the radial steering bogies can be applied [8,9]. Figure 3 shows the schematic diagrams of the self-steering and controllable radial steering bogies.

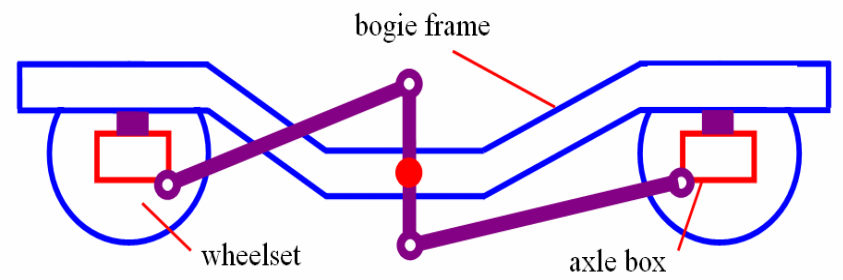

(a) self-steering bogie

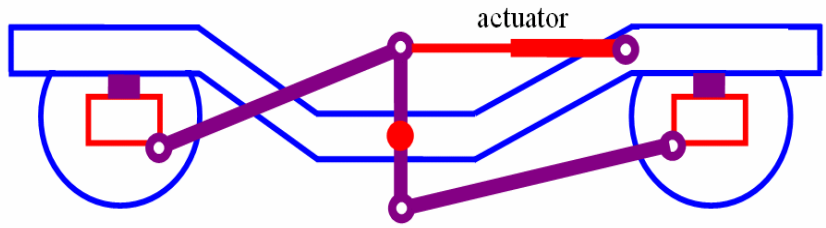

(b) controllable steering bogie

Fig.3 Self-steering and controllable steering bogies

For the forced-steering bogie, the vertical lever of the self-steering mechanism can be prolonged to connect with the carbody, and an appropriate steering gain of the lever is designed. For the controllable radial steering bogie, an electromechanical actuator can be mounted between the self-steering mechanism and the bogie frame. The reference control signal of the actuator can be obtained from several ways, such as the yaw angle between the carbody and bogie frame, yaw angle between the two adjacent carbodies, tilting control signal and train speed. Here, the yaw angle between the front carbody and the leading bogie frame is adopted. This angle signal should be filtered at first and the cutoff frequency should be as low as possible to get the smooth signal for the control in the case of not causing too long time delay. In order to keep the wheelset to take radial position on any radius curved track, the wheelset should be forced to yaw an angle $\psi_{w}$ that satisfies the following relation:

$$
\psi_{w}=\frac{L_{b}}{L_{c}} \psi_{c b}
$$

where $\psi_{c b}$ is the relative yaw angle between carbody and bogie frame, $L_{b}$ and $L_{c}$ are the half of wheelbase and half of bogie distance respectively. Thus, the displacement of the actuator should be as below:

$$
d_{a}=\frac{L_{s} L_{b}}{L_{c}} \psi_{c b}
$$


where $L_{s}$ denotes half of the lateral distance between the steering mechanisms on the bogie left and right sides.

Figure 4 represents the bogie controllable steering properties on curve $R=600 \mathrm{~m}$ at speed $140 \mathrm{~km} / \mathrm{h}$. It is seen that the filtered control signal is quite smooth which is advantageous for the bogie steering control. Although small time delay due to filtering exists, good radial steering can be still realized for the front bogie on circular curved track. Then the later bogies can easily maintain radial positions when passing through curves by giving a corresponding time delay to the actuator control according to the bogie wheelbase and bogie distance.

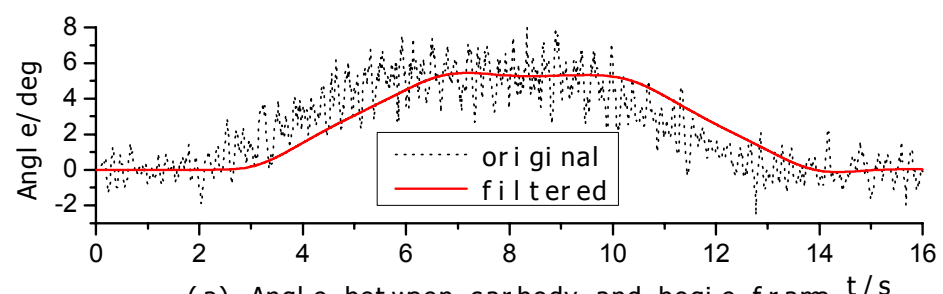

(a) Angl e between carbody and bogi e frame t/s
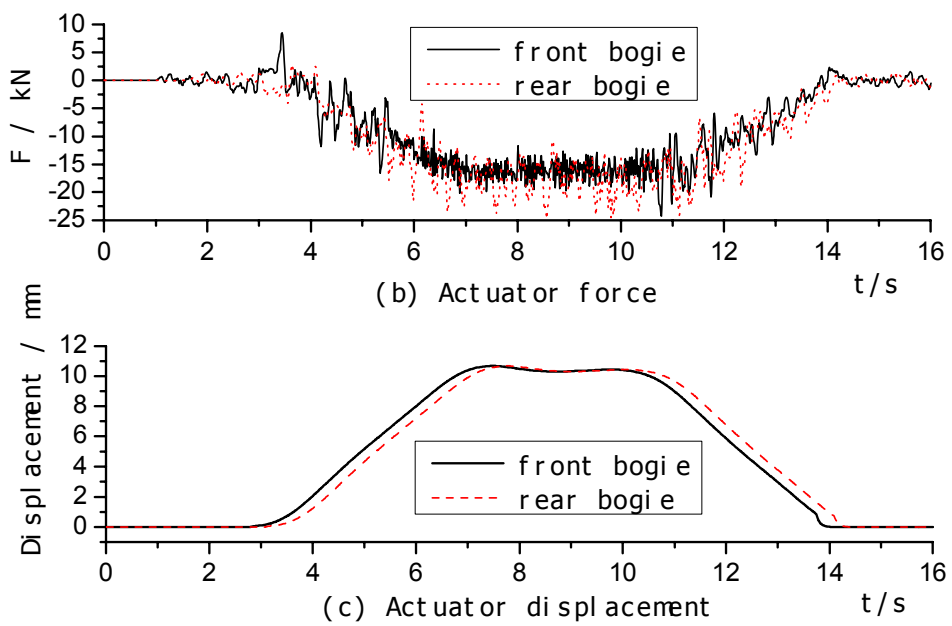

Fig.4 Controllable steering

\section{Carbody tilting control}

A lateral acceleration sensor and a gyroscope are located on the leading bogie of the first car to obtain the tilting signals for each car, and an electromechanical actuator is mounted on each bogie. Because of the filtering and processing of the measured signals, the time delay exists for the tilting control of each car. The control signal of the first car has longer time delay that can be partly compensated by using the gyroscope signal and forecasting methods. The time delay for the second or third car is further reduced and the control signal is getting smoother. The tilting control system is depicted by Fig.5. The tilting train can negotiate curves with a speed about $30 \%$ higher than the conventional train.

The state space equations of the actuator is as below

$$
\left\{\begin{array}{l}
\dot{x}_{1}=\left(U_{m}-K_{e} x_{3}-R_{m} x_{1}\right) / L_{m} \\
\dot{x}_{2}=x_{3} \\
\dot{x}_{3}=\left(K_{t} x_{1}-B_{e} x_{3}-T_{f} / F\right) / J
\end{array}\right.
$$

where $x_{1}$-motor current

$\mathrm{x}_{2}$ - rotary angle of motor axle

$\mathrm{x}_{3}$ - angular velocity of motor axle

$U_{m}$-input voltage 
$K_{e}$-inducted voltage constant

$R_{m}$-armature resistance

$L_{m}$ - armature inductance

$B_{e}$ - equivalent damping coefficient

$T_{f}$-load torque

$K_{t}$ - constant of motor torque

$J$-equivalent moment of inertia of motor

$F$ - actuator force

The displacement $k_{2} x_{2}$ of the actuator is taken as the output feedback control, here $k_{2}$ denotes the transmission ratio between the motor rotary angle and leading screw displacement. The transfer function is written as

$$
G(s)=\frac{9.7843 \times 10^{3}}{s^{3}+6.6066 \times 10^{2} s^{2}+2.1725 \times 10^{6} s}
$$

For the above transfer function, the motor voltage is the input and the actuator displacement is the output. According to the classical transfer function of the servo motor, the effect of the load torque is not considered in the transfer function. But the differential equation (4) is adopted for the simulation in which the load torque determined through vehicle dynamics calculation is considered.

For the carbody tilting control, the $P I$ and $H \infty$ robust controllers are designed. Because the $I$ value (1.0 used) is much smaller than the $P$ value in the $P I$ controller, and has less influence on the dynamic response, only the influence of $P$ with certain proportion coefficient is discussed in this paper. The standard $H \infty$ robust control diagram is shown as Fig.6, in which $w$ is the tilting control signal, $v$ the feedback disturbance, $\left(w_{1}, w_{2}, w_{3}\right)$ the controlled output variables, $\left(W_{1}, W_{2}, W_{3}\right)$ the performance weighting functions, and $K(s)$ the controller.

The design criteria for the tilt control are that the tilting system should have rapid response and small tilting angular acceleration. In order to reduce the influence of the disturbance on the control performance and increase the response ability, appropriate $W_{l}$ value should be selected to increase the anti-disturbance ability in low frequencies. In order to reduce the possible large tilting angular acceleration caused by the control signal, and meanwhile to restrain the load effect in high frequencies, the $W_{3}$ with high frequency restraint is adopted. Since there is no particular requirement for the actuator input signal, a relative small $W_{2}$ can be utilized which only needs to satisfy the existence condition of the robust stabilizing of the controller. Therefore, the weighting functions are given finally as

$$
\left\{\begin{array}{l}
W_{1}(s)=\frac{\alpha(s+30)}{s+2} \\
W_{2}(s)=1.0 \times 10^{-3} \\
W_{3}(s)=0.05(s+10)
\end{array}\right.
$$

The discrete control with frequency $250 \mathrm{~Hz}$ is used. Thus, the designed discrete robust controller is

$$
K(z)=\frac{1.794 \mathrm{z}^{-1}-3.817 \mathrm{z}^{-2}+2.392 \mathrm{z}^{-3}-0.494 \mathrm{z}^{-4}+0.126 \mathrm{z}^{-5}}{1-3.036 \mathrm{z}^{-1}+3.267 \mathrm{z}^{-2}-1.488 \mathrm{z}^{-3}+0.322 \mathrm{z}^{-4}-0.064 \mathrm{z}^{-5}}
$$




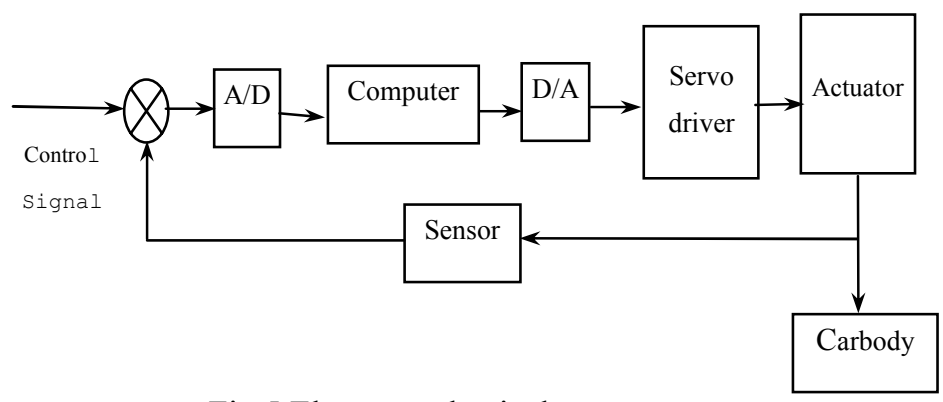

Fig.5 Electromechanical servo system

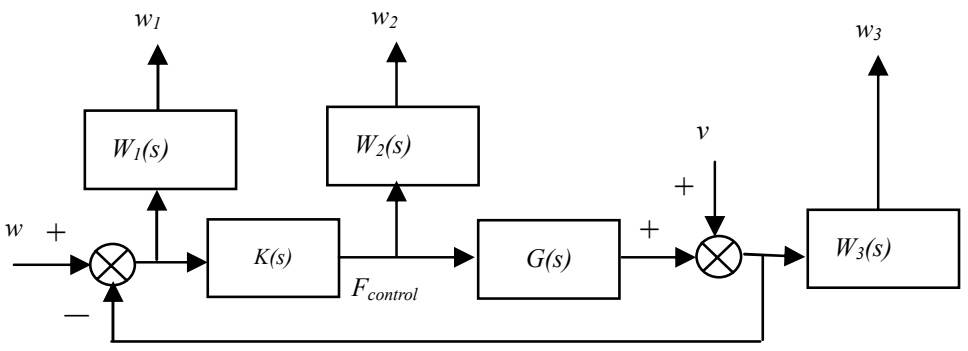

Fig.6 $H \infty$ robust control

Figure 7 illustrates the unit step responses of the tilting system with $P$ control and $H \infty$ robust control. It is seen that the robust control performance is better than the $P$ control, but the response time of robust control is little longer than the one of $P$ control.
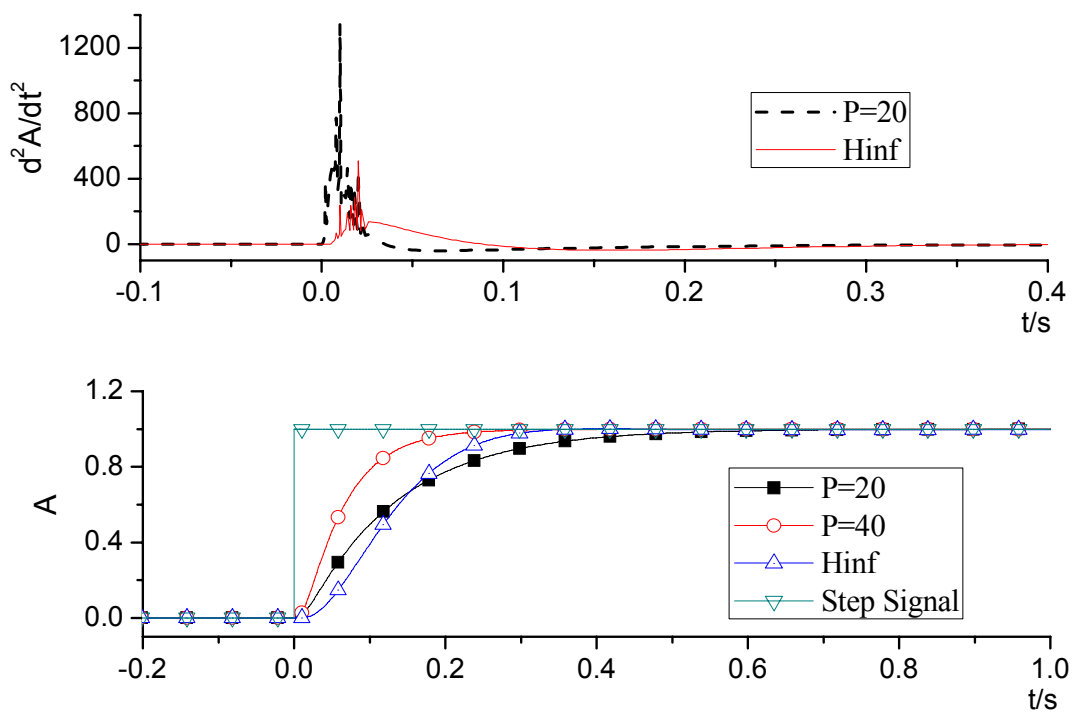

Fig.7 Unit step response of tilting system

In order to reduce the time delay of the signal filtering, the neural network forecast and linear forecast methods are adopted to obtain the tilting control signals of the front car. Through comparison and analysis, the linear neural network with three layers and single output is applied. According to the simulation, the time of the filtered tilting control signal is moved up and taken as the learning objective for the neural network. The original signal together with the filtered signal is regarded as the input of the neural network. While for the linear forecast, the former tilting signals are extrapolated linearly to obtain the tilting signal at the current moment. 


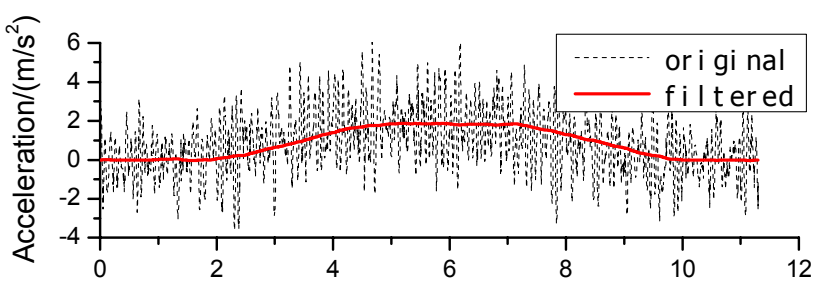

(a) Lateral Acc. of the 1st Frame

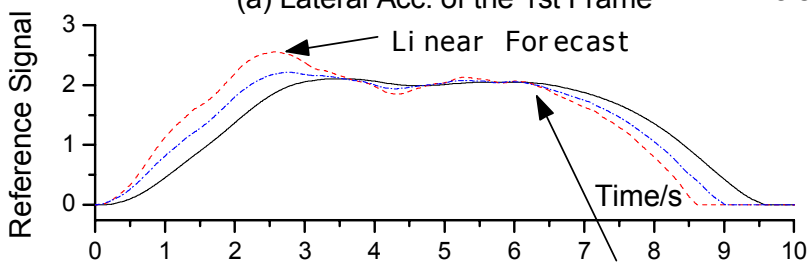

(b) Tilting Control Ref. Signal ........1st car

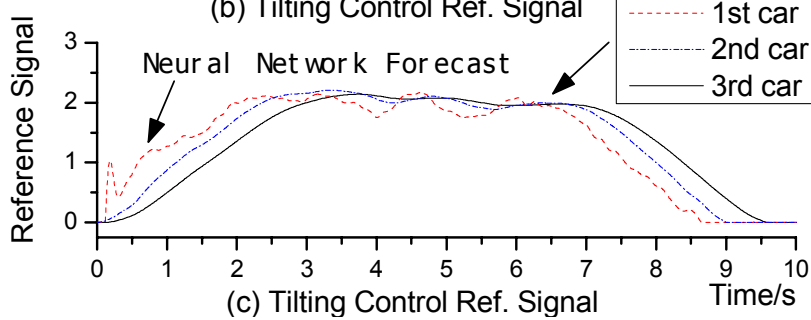

Fig.8 Lateral acceleration and control reference signal

The curved track information is obtained from the filtered signals of the gyroscope on the leading bogie frame of the first car, and the tilting control signal is based on the filtered lateral acceleration of the sensor on the leading bogie. Figure 8 shows the control reference signals of the first car which are based on the linear forecast and neural network forecast with considering $70 \%$ compensation of the unbalanced lateral acceleration. Figure 9 and 10 illustrate the tilting behavior with linear forecast and neural network forecast, in which the red dash lines represent the ideal tilting angles. It is seen from Fig.9 and 10 that the linear forecast method has a larger time delay than the neural network forecast method.
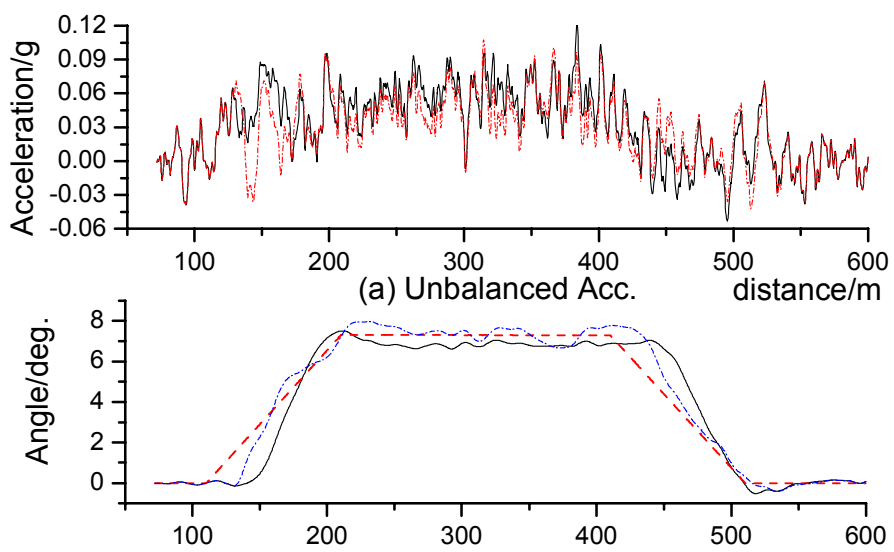

(b) Tilting Angle of Carbody distance/m

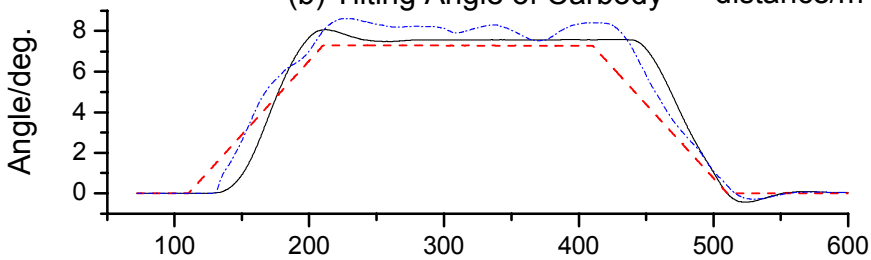

(c) Tilting Angle of Bolster distance/m

linear forecast noural network forecast

Fig.9 Tilting behaviour of 1 st car with $H \infty$ control 


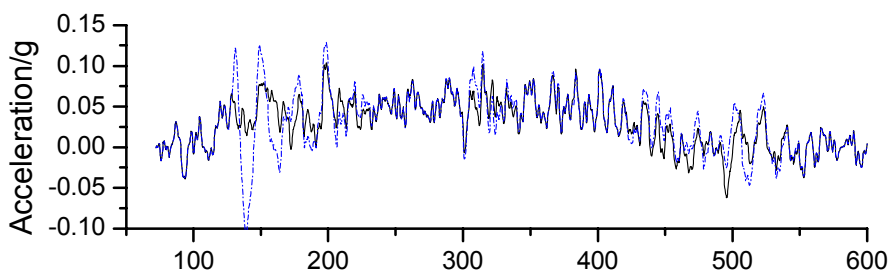

(a) Unbalanced Acc. distance/m

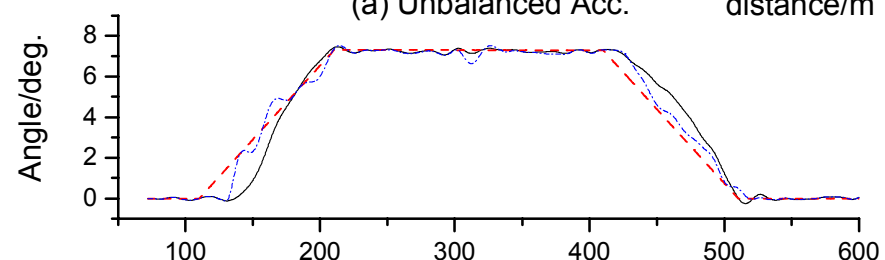

(b) Tilting Angle of Carbody distance $/ \mathrm{m}$

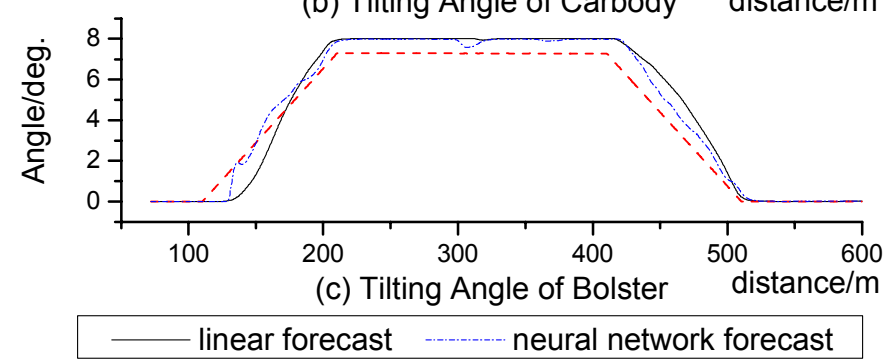

Fig.10 Tilting behaviour of 1 st car with $P$ control

\section{Curving behavior of tilting train}

Figure 11 illustrates the tilting angles of the carbody and the bolster when the train negotiates curve $R=600 \mathrm{~m}$ with speed $140 \mathrm{~km} / \mathrm{h}$. The ideal tilting angle of the carbody is defined as the angle when $70 \%$ of the unbalanced lateral acceleration of carbdoy compensated. It is known that the bolster and carbody are able to follow the ideal tilting angles, but the carbody tilting angle is less than the bolster tilting angle due to the secondary suspension deflection. The unbalanced lateral acceleration of carbody is shown as Fig.12, which indicates that the unbalanced acceleration exceeds $2.0 \mathrm{~m} / \mathrm{s}^{2}$ for carbody none tilting, and it can be reduced significantly for carbody tilting.

The nominal power of actuator is defined as the product of actuator force and velocity. Figure 13 shows that the $P=40$ control has largest actuator power with large fluctuation, and the robust control has smallest power, which means that the control method will have strong influence on the actuator and will further affect the tilting behavior.

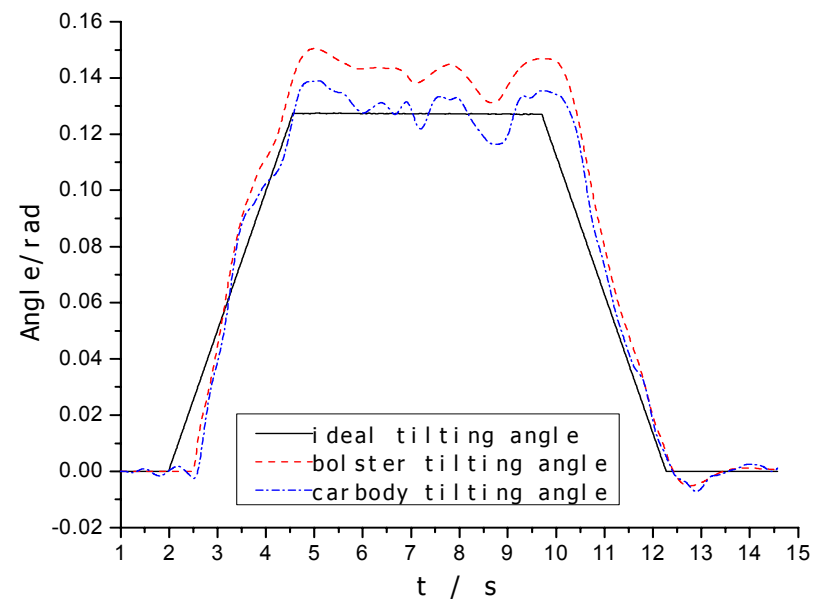

Fig.11 Tilting angles of bolster and carbody 


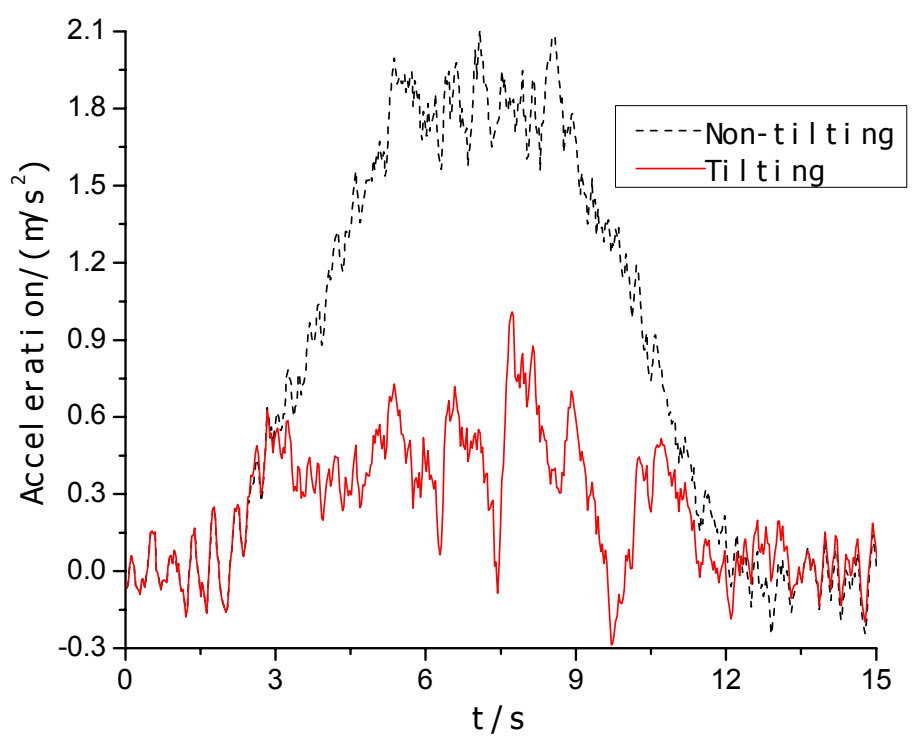

Fig.12 Carbody unbalanced lateral acceleration
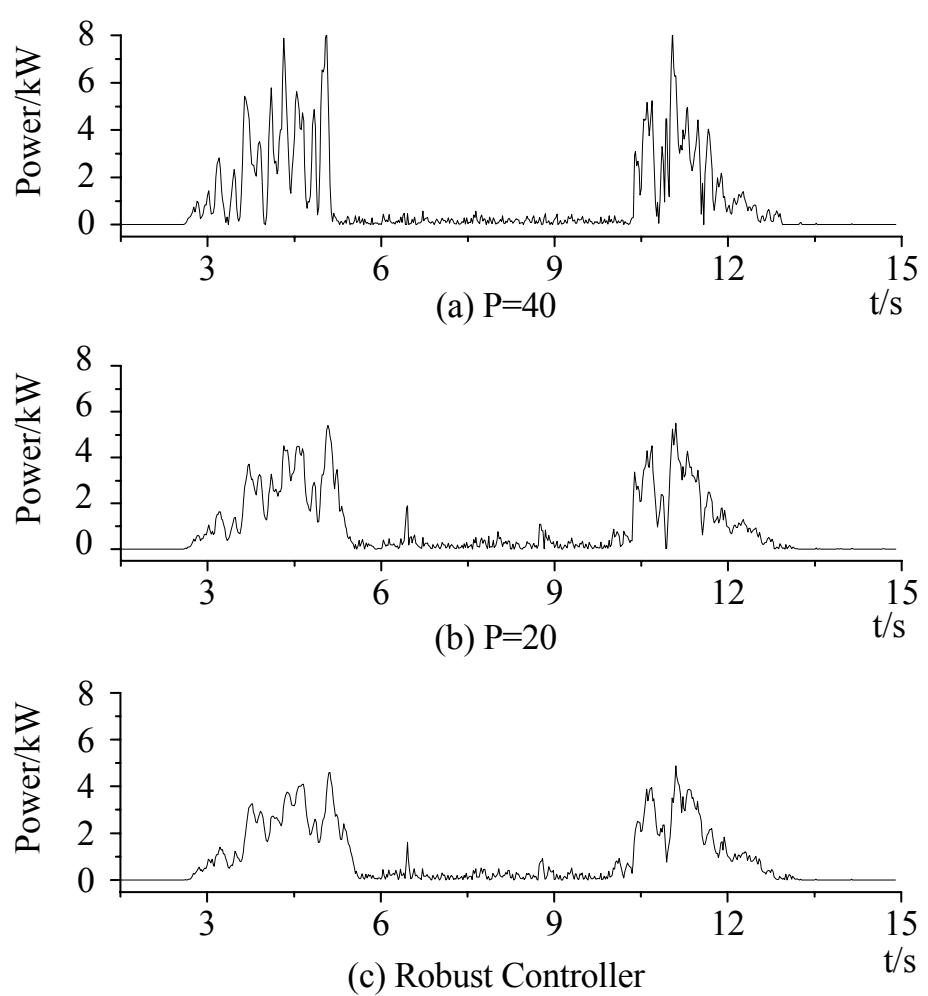

Fig.13 Nominal power of actuator

Figure 14 shows the curving behavior comparison of the first car of the tilting train and conventional train. For the curving simulation, the track curve radius $R$ equals to $400 \mathrm{~m}$ to $1400 \mathrm{~m}$ with corresponding speed from 80 to $130 \mathrm{~km} / \mathrm{h}$ for the conventional train and $30 \%$ speed higher for the tilting train. It is seen from the results that the outer wheel lateral force $Q$, derailment coefficient $Q / P$ and wheel unloading ratio $\triangle P / P$ of the tilting train are larger than the conventional train due to the tilting train operating with higher speeds. 

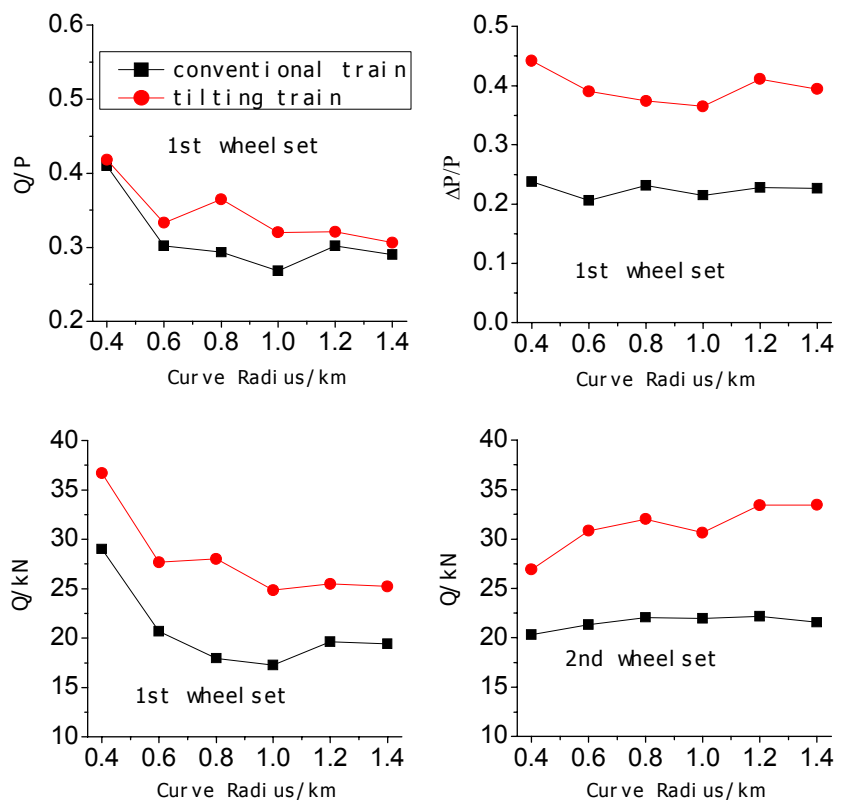

Fig.14 Curving behavior comparison

The wheel-rail interaction force and wear work for different types of bogies are calculated by numerical simulation for the tilting train. Figure 15 and 16 demonstrate the results for the first car on curve $R=300 \mathrm{~m}$ and $R=800 \mathrm{~m}$ at different speeds. In the figures, the wheelset lateral force and derailment coefficient are the maximum values, and the wheel/rail wear work done is the mean value for the whole vehicle on the simulation distance. It is seen that the lateral force, $Q / P$ and wear work of different radial steering bogies are all smaller than the conventional bogie, but the forced and controllable steering bogies have better curving behavior compared with the self-steering and conventional bogies, especially on small curves as $R=300 \mathrm{~m}$.
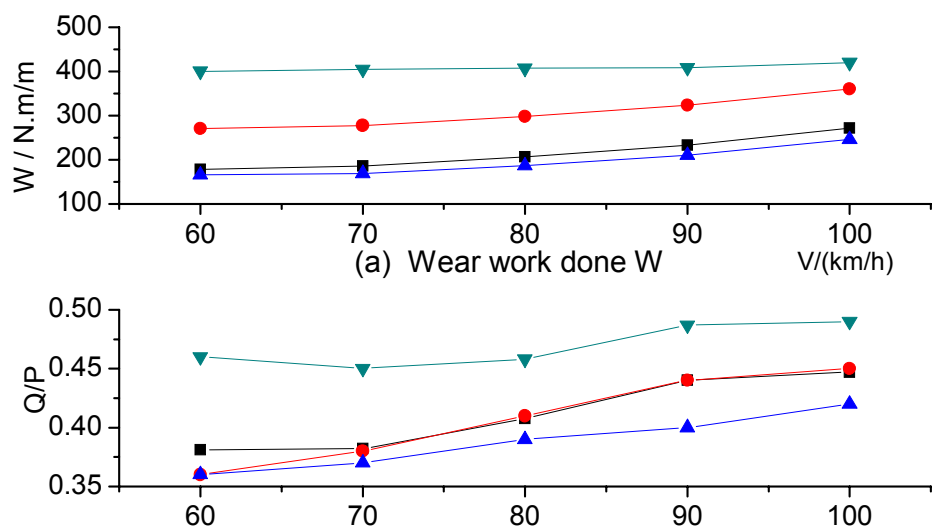

(b) Derailment coefficient Q/P V/(km/h)

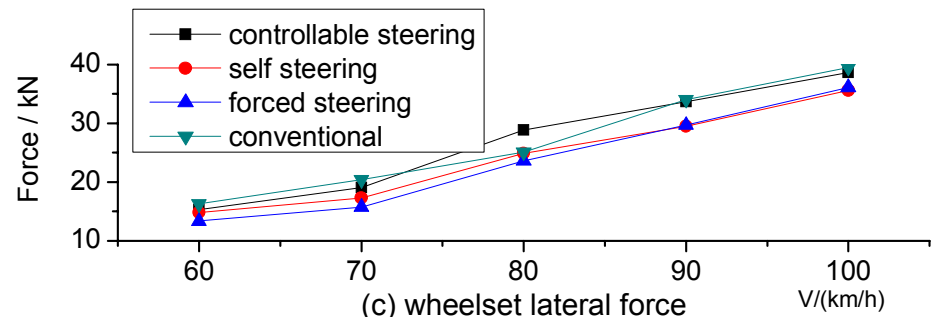

Fig.15 Curving behavior on R300m 


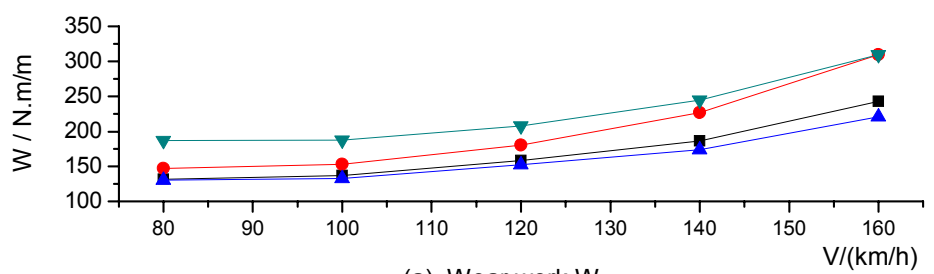

(a) Wear work W

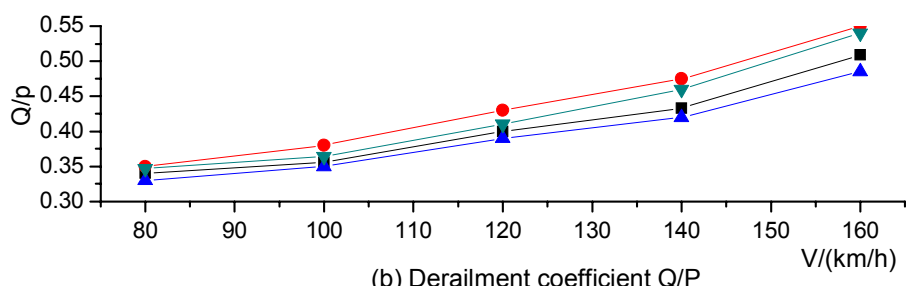

(b) Derailment coefficient Q/P

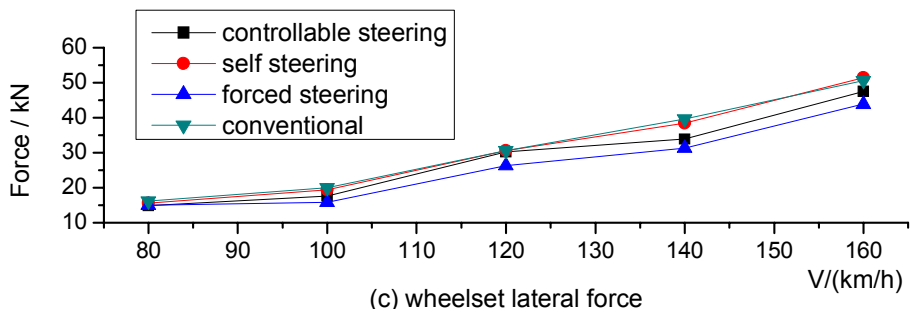

Fig.16 Curving behavior on R800m

\section{Conclusions}

This paper mainly deals with the computer simulation of the dynamic curving performance of the tilting train, and the following conclusions can be drawn:

(1) The application of carbody tilting technology is the most efficient way to raise train speed during curve negotiation. The main function of the tilting train is to reduce the unbalanced lateral acceleration felt by passengers through inclining carbody towards inside of the curve.

(2) In order to achieve better curving performance and running safety at the same time, radial steering bogies for the tilting train should be taken into consideration.

(3) The nonlinear mathematical model for the titling train composed of three cars is built by considering the coupler force, traction force and resistant force. The nonlinear factors included in the model are the nonlinear wheel/rail contact geometry and interaction forces, nonlinear coupler force and nonlinear secondary suspension forces.

(4) The lateral acceleration of the leading bogie of the first car is used as the control signal of the tilting system. For the carbody tilting control, the $P I$ and $H \infty$ robust controllers are designed. The time delay caused by the measuring and control system is compensated by using the gyroscope on the leading bogie frame of the first car, and the linear forecast and neural network forecast methods.

(5) Finally, the curving behavior of the tilting train including the carbody tilting angle, unbalanced acceleration and wheel/rail interactions are investigated, especially the wheel/rail force, wear work done and derailment safety of the self-steering, forced-steering, controllable steering and conventional bogies are compared.

\section{Acknowledgement}

The authors would like to thank the National Natural Science Foundation of China (No.50675182) and the National Innovation and Research Teams Foundation (No.50821063) for funding this research. 


\section{References}

(1) Goodall, R. M., Pacejka, H. B. and Sharp, R. S.: Active Railway Suspension: Implementation Status and Technological Trends, Vehicle System Dynamics, Vol.28, 87-117 (1997).

(2) Bruni, S., Goodall, R. M., Mei, T. X. and Tsunashima H.: Control and Monitoring for Railway Vehicle Dynamics, Vehicle System Dynamics, Vol.45, 743-779 (2007)

(3) Harris, N. R., Schmid, F. and Smith, R. A.: Introduction: Theory of Tilting Train Behaviour., Journal of Rail and Rapid Transit, Vol.212, 1-6 (1998).

(4) Cheli, F., Diana, G. and Resta, F.: Numerical Model of a Tilting Body Railway Vehicle Compared with Rig and on Track Tests, Vehicle System Dynamics, Vol.29, 417-442 (2001).

(5) Zolotas, A.C., Goodall, R.M. and Halikias, G.D.: New Control Strategies for Tilting Trains, Vehicle System Dynamics, Vol.37, 171-182 (2002).

(6) Huber, B. H.: The Bogie-Based Tilt Option - Simplicity and Flexibility, Journal of Rail and Rapid Transit, Vol.212, 19-32 (1998).

(7) Schmid, F.: Control and Operation of Tilting Train Services, Journal of Rail and Rapid Transit, Vol.212, 61-72 (1998).

(8) Mei, T. X. and Goodall, R. M.: Model Controllers for Active Steering of Railway Vehicles with Solid Axle Wheelsets, Vehicle System Dynamic, Vol.34, 301-322. (2000).

(9) Garcia, J. F., Olaizola, X., Martin, L. M. and Gimenez, J. G.: Theoretical Comparison between Different Configurations of Radial and Conventional Bogie, Vehicle System Dynamic, Vol.33, 233-259 (2000). 